\title{
A interface entre Saúde Mental e Vulnerabilidade Social
}

\section{The interface between Mental Health and Social Vulnerability}

\section{La interfaz entre Salud Mental y Vulnerabilidad Social}

\author{
Carolina Francielle Tonin ${ }^{1}$ \\ Tatiane Muniz Barbosa ${ }^{2}$
}

RESUMO: Esta pesquisa objetivou conhecer o entendimento dos profissionais da Atenção Primária a Saúde, de uma unidade de saúde num município do interior de SC, sobre a interface entre Saúde Mental e Vulnerabilidade Social. O projeto de pesquisa foi apreciado pelo CEP/UNIPLAC, com parecer aprovado $\mathrm{n}^{0}$ 721.689. A coleta de dados aconteceu por meio de entrevista semiestruturada, com aplicação do TCLE, com dez profissionais de equipes de ESF e NASF. Os dados, tratados por meio de análise de conteúdo, indicaram que os profissionais de saúde compreendem a saúde mental integrante da saúde "geral" e acreditam que a falta de educação formal, desconhecimento dos serviços da rede, estresse e desemprego são fatores que influenciam no sofrimento psíquico. Quanto à vulnerabilidade social, relacionaram com a questão econômica e a descreveram como falta de condições de saneamento básico, moradia, escolaridade e autonomia. Os profissionais veem relação entre Saúde Mental e Vulnerabilidade Social, pois, a exposição das pessoas a situações de vulnerabilidade social e risco de sofrimento psíquico não é resultante apenas de aspectos individuais, mas, coletivos. Percebe-se que o cuidado em saúde mental aparece fragmentado; contudo, os profissionais de saúde têm buscado considerar novas estratégias para lidar com esta, bem como em relação à vulnerabilidade social, através de um trabalho multiprofissional, voltado às necessidades das famílias. Ainda é evidente uma prática assistencialista, quando se faz necessário criar condições para que estas famílias se conscientizem e se tornem protagonistas de suas vidas.

Palavras-chave: Vulnerabilidade Social, Atenção Primária à Saúde, Saúde Mental.

\begin{abstract}
This research aimed to know how the professionals of Primary Health of a health center, in the interior of the municipality of SC, understand the interface between social vulnerability and mental health. The research project was valued by CEP/UNIPLAC, endorsed by No 721.689 . Thereby, the data collection took part by means of semi-structured interview, previously scheduled and with the application of the TCLE, ten professionals were interviewed both in ESF and NASF. For data analysis the content method analysis was used. All obtained data pointed that health professionals have comprehended mental health integral from "general" health, and so they believe the lack of formal education, ignorance of the network services, stress, unemployment, are coefficients weighting on the psychological distress forthcoming. Concerning social vulnerability, respondents related it to the economic situation, also described it as a lack of sanitation, dwelling, education and autonomy. The professionals see a relationship between Mental Health and Social Vulnerability because the exposure of people in conditions of social vulnerability and risk of

1 Possui graduação em Psicologia (Bacharel) pela Universidade do Planalto Catarinense (2012); especialização em Residência Multiprofissional em Saúde da Família e Comunidade (2015). Experiência profissional na área de Saúde da Família. Prefeitura Municipal de Brunoópolis. Brunópolis, Santa Catarina, Brasil. E-mail: carolf.tonin@gmail.com 2 Graduação em Psicologia pela Universidade do Vale do Itajaí (2002). Doutora em Saúde Coletiva pela Universidade Federal de Santa Catarina (2015). Universidade do Planalto Catarinense (UNIPLAC). Santa Catarina, Brasil. E-mail: tatianemb.tmb@gmail.com
\end{abstract}

ISSN 1982-8829 Tempus, actas de saúde colet, Brasília, 11(3), 50-68, 2017 - Epub mar, 2018 
psychological distress, it's not only a result of individual aspects but also collective. Throughout this research it is seen that mental health care turns up broken, although health professionals have been seeking for new strategies to deal with the mentioned care, as well as the social vulnerability, through a multidisciplinary work, aimed at family needs. A welfare exercise is evident, when the creation of proper conditions would be necessary so that these families could become aware and the leading figure of their lives.

Keywords: Social vulnerability, Primary Health Care, Mental Health.

RESUMEN: Esta investigación objetivou conocer la comprensión de los profesionales de la Atención Primaria de Salud, de una unidad de salud en un município del interior de SC, sobre interfaz entre Salud Mental y Vulnerabilidad Social. El proyecto de investigación fue apreciado por el CEP/UNIPLAC, con parecer aprobado $\mathrm{n}^{\circ}$ 721.689. La recolección de datos aconteció por medio de entrevista semiestruturada, con aplicación del TCLE, con diez profesionales de equipos de ESF y NASF. Los datos, tratados por medio de análisis de contenido, indicaron que los profesionales de salud comprenden la salud mental integrante de la salud y creen que la falta de educación formal, desconocimiento de los servicios de la red, estresse y desempleo son factores que influéncian en el sufrimiento psíquico. En cuanto la vulnerabilidad social, relacionaron con la cuestión económica y a describieron como falta de condiciones de saneamiento básico, vivienda, escolaridad y autonomia. Los profesionales veem relación entre Salud Mental y Vulnerabilidad Social, pues, la exposición de las personas la situaciones de vulnerabilidad social y riesgo de sufrimiento psíquico no es resultante sólo de aspectos individuales, pero, colectivos. Se percibe que el cuidado en salud mental aparece fragmentado; pero, los profesionales de salud tienen buscado considere nuevas estrategias para lidar con esta, así como en relación a la vulnerabilidad social a través de un trabajo multiprofissional, vuelto a las necesidades de las familias. Aún es evidente una práctica assistencialista, cuando se hace necesario crear condiciones para que estas familias se conscientizem y se hagan protagonistas de sus vidas.

Palabras clave: Vulnerabilidad social, Atención Primaria de Salud, Salud Mental.

\section{INTRODUÇÃO}

Com as vivências enquanto psicólogas e como residente em uma Unidade de Saúde da Família-escola, junto à equipe do Núcleo de Apoio a Saúde da Família (NASF) observou-se que a maioria dos casos encaminhados à equipe se referia a Saúde Mental e estes estavam concentrados, sobretudo, em bairros periféricos da cidade. Espaços onde as pessoas estão sob exposição ao risco social, ressaltam Batista e Carvalho-Silva ${ }^{1}$, posto que estão ameaçadas pela insalubridade, condições de moradia e os próprios laços sociais, apesar dos esforços dos moradores e organizações do território, encontram-se esgarçados.

Acredita-se que ainda há segregação urbana (entre classes) e prevalecem mecanismos de exclusão em relação aquilo que não se enquadra no "padrão de normalidade" da sociedade. Portanto, mesmo não existindo instituições manicomiais, as pessoas em sofrimento psíquico permanecem sendo excluídas da sociedade e com recursos precários para obterem qualidade de vida.

Percebeu-se poucas ações e construções de projetos terapêuticos, na Atenção Primária à Saúde (APS), que auxiliassem no cuidado, prevenção e promoção em Saúde Mental e reduzissem 
o preconceito e a segregação da "loucura". Principalmente porque com práticas mais resolutivas, dar-se-ia um passo a frente em relação à Saúde Mental e à vulnerabilidade social.

Diante disso, surgem questionamentos acerca do impacto que a Vulnerabilidade Social pode ter no surgimento/agravo de problemas de ordem mental. De acordo com Bezerra $\mathrm{Jr}^{2}{ }^{2}$, o sofrimento psíquico é socialmente forjado pelas condições de miséria e sem alternativas políticas as pessoas acabam adoecendo como estratégia de sobrevivência.

A palavra vulnerabilidade tem origem latina e significa "ferida", assim, pessoas vulneráveis são aquelas suscetíveis a serem "feridas" . O conceito iniciou na área dos Direitos Humanos e foi incorporado à Saúde através de estudos sobre a AIDS ${ }^{4}$. A Vulnerabilidade Social e a Saúde Mental não são conceitos recentes, por isso assumem diferentes significados, complexos e multifacetados. Entretanto, habitualmente são conceitos reduzidos a outras expressões, o primeiro à situação econômica (pobreza), enquanto o segundo as psicopatologias.

Afirmam Guareschi; Bernardes ${ }^{4}$, a vulnerabilidade se desenvolve quando existe um aumento na carência, tanto de informações quanto uma despreocupação com as pessoas expostas ao perigo, sem contar a inacessibilidade aos serviços básicos e a falta de confiança na sustentação de estratégias de saúde. Portanto, a vulnerabilidade social abrange a precariedade em diferentes segmentos considerados de "necessidade básica" e ainda questões de organização política, etnia, orientação sexual, dentre outras.

A vulnerabilidade social se aproxima do conceito ampliado de saúde, preconizado pelo SUS, demonstrando que ambos ocorrem de maneira diferente em cada território e com cada pessoa/ família. Nessa acepção, Vulnerabilidade Social diz respeito às políticas públicas, particularmente quando estas entendem o conceito de Saúde como macropolítico e plural ${ }^{4,6,7}$.

Pontua-se ainda, que o agravo nas questões de Vulnerabilidade Social e Saúde Mental, refere-se à falta de conscientização ${ }^{3}$ dos sujeitos envolvidos e de uma política assistencialista que prevalece no País. Além das pessoas vivenciarem espaços estigmatizadores e vulneráveis, essas se percebem diferentes (em razão do sofrimento psíquico) e assumem determinadas posições de sujeito; pois, tornam-se vulneráveis aos olhos dos outros, em diferentes esferas de suas vidas, que os enxergam como incapacitados de exercerem outros papéis sociais, fazer escolhas e tomar decisões. Esta "visão" é internalizada por aqueles que sofrem psiquicamente e isso dificulta a alteração da realidade dos mesmos ${ }^{4}$.

Diante disso, este estudo buscou compreender o entendimento dos profissionais da APS, de uma USF do município do interior de SC, acerca da interface entre Saúde Mental e Vulnerabilidade Social. Para isso, o contexto da pesquisa se pautou na metodologia qualitativa, com aplicação de 3 O termo conscientização, utilizado e explicado por Freire ${ }^{8}$, implica no "ato" de ultrapassar a esfera de apreensão da realidade para chegar à esfera crítica, onde a realidade se dá.

ISSN 1982-8829 Tempus, actas de saúde colet, Brasília, 11(3), 50-68, 2017 - Epub mar, 2018 
entrevistas semiestruturadas a 10 profissionais de saúde que atuam em uma USF, assumindo-se que ao compreender a relação entre Saúde Mental e Vulnerabilidade Social se provoca reflexão e construção de novas concepções e práxis no cuidado em saúde.

Mesmo com as significativas mudanças oriundas da Reforma Psiquiátrica (1970), sobretudo em relação ao modo de cuidado das pessoas em sofrimento psíquico, o processo de autoafirmação exige mais do que a mudança de nomenclaturas dos serviços. Para alcançar transformações no saber-fazer - superando o modelo biomédico, dado que ainda há práticas que medicalizam a vida são necessários espaços criativos e conjuntos interdisciplinares e transdisciplinares ${ }^{9}$.

A relevância desta pesquisa está na desconstrução de estigmas cristalizados pela sociedade e campos de saber que auxiliam na legitimação destes. Afinal, as pessoas em sofrimento psíquico precisam se reconhecer como sujeitos ativos, produzirem-se em diferentes esferas da vida e não permanecerem "portadores de marcas identitárias"4 em relação ao sofrimento psíquico e à Vulnerabilidade Social.

Através da mobilização dos profissionais da APS estas mudanças podem se corroborar, pois são estes que investem nas potencialidades dos sujeitos/famílias, orientando-os e os preparando para lidar com as limitações referentes aos problemas de ordem mental, principalmente, quando em situações de Vulnerabilidade Social. Assim, estar-se-á contribuindo para que os sujeitos se percebam e se produzam de maneiras diferentes, destaca Bezerra $\mathrm{Jr}^{2}$, promover-se-á saúde considerando o desenvolvimento das condições de vida justas e equânimes, conforme os princípios do SUS.

\section{PERCURSO METODOLÓGICO}

O presente estudo, qualitativo, foi apreciado pelo Comitê de Ética em Pesquisa da UNIPLAC, com parecer favorável ( $\mathrm{n}^{\circ}$ 721.689). A pesquisa foi realizada em um município do interior de SC, com população estimada de 160 mil habitantes, segundo o Instituto Brasileiro de Geografia e Estatística (IBGE) em 2013.

Neste município existem 26 unidades de saúde em área urbana e 2 em área rural e há 4 equipes de NASF, modalidade tipo I, as quais podem acompanhar de 5 a 9 equipes de ESF. Estas equipes NASF são compostas por 4 categorias profissionais: psicólogo, nutricionista, assistente social, fisioterapeuta e/ou educador físico. Salienta-se que 3 USF da área urbana funcionam como "unidades-escola", onde atuam os residentes de um Programa de Residência Multiprofissional em Saúde da Família e Comunidade (RMSFC) - parceria entre SMS e Universidade.

A pesquisa foi realizada em uma USF da área urbana, bairro periférico da cidade, que apresenta equipes de Saúde da Família e Saúde Bucal e é acompanhada por uma das equipes de NASF. Para escolha do local do estudo se considerou que a referida USF funciona como "unidade- 
escola" da RMSFC, tem equipe de matriciamento do NASF e a pesquisadora não tinha vínculo com as equipes dessa USF.

A USF participante desse estudo possui 3 equipes de ESF, 2 de Saúde Bucal e 1 de NASF, ou seja, a escolha desse local de pesquisa buscou privilegiar a atuação multiprofissional na APS para conhecer o entendimento desses profissionais sobre a interface entre Saúde Mental e Vulnerabilidade Social.

O universo de participantes foi composto por 10 profissionais de saúde, definidos a partir dos seguintes critérios: maior tempo de atuação na APS; um representante de cada categoria profissional (médico, enfermeiro, técnico de enfermagem, agente comunitário de saúde, dentista, técnico em higiene dental, psicólogo, fisioterapeuta, assistente social e nutricionista) e estar atuando na USF durante o período de coleta de dados.

A coleta de dados se deu através de entrevistas semiestruturadas, individuais e agendadas previamente na USF. Optou-se pela entrevista semiestruturada porque essa técnica permite falas espontâneas do participante e valoriza a presença do pesquisador. Questionamentos básicos, propostos em teorias e hipóteses da pesquisa, dão espaço para outras questões, resultado de novas hipóteses e variáveis que surgem a partir das falas dos entrevistados ${ }^{10}$. Aplicou-se o TCLE e as entrevistas foram registradas via gravação em áudio com autorização dos participantes, em respeito à Resolução 466/12 do CNS.

$\mathrm{Na}$ análise dos dados utilizou-se a técnica de análise de conteúdo temática, realizou-se leitura flutuante, decompuseram-se as falas, destacaram-se as unidades de registro e se construíram categorias temáticas ${ }^{11}$.

\section{RESULTADOS E DISCUSSÃO DOS DADOS}

As entrevistas com os profissionais de saúde tiveram duração média de 15 a 30 minutos cada uma e para preservar a identidade dos profissionais se utilizou a letra $\mathrm{S}$ (sujeito) e a numeração de 1 a 10 para representá-los. Os entrevistados apresentavam idade média de 30 anos; dos 10 entrevistados, 9 eram mulheres. Tal dado é interessante, pois, mesmo com as mudanças que ocorrem nos padrões culturais, nos valores e no papel social que a mulher exerce nos dias atuais, ainda há predomínio destas em trabalhos voltados ao cuidado. Pontuam Lopes e Leal ${ }^{12}$ essa "naturalização" da mulher como cuidadora decorre de um processo sociohistórico onde a mulher exercia o cuidado doméstico das crianças, doentes e idosos, além de apresentar marcas de ordens religiosas.

O tempo de atuação dos profissionais na APS variava entre 2 a 13 anos. Dos pesquisados, 4 exercem outras atividades além da sua profissão, sendo todos estes docentes. É significativa a questão de mais de um vínculo empregatício, uma vez que se trata da realidade de muitos profissionais brasileiros. Trazem, Fernandes, Miranzi ${ }^{13}$, em sua pesquisa que $33 \%$ dos enfermeiros da ESF, do 
triângulo mineiro, possuíam outros empregos. Percebe-se que esse movimento ocorre em função do vínculo empregatício temporário, carga horária excessiva e remuneração insatisfatória. Estas características podem levar os profissionais a se sentirem inseguros e insatisfeitos com o trabalho, interferindo na qualidade do atendimento prestado e na qualidade de vida dos mesmos.

\subsection{Saúde vs. Saúde Mental: segregar não é preciso}

As falas dos entrevistados, de modo geral, indicam que a Saúde Mental é compreendida como uma área de atuação "fragmentada" da Saúde "geral" e que trata, especificamente, de pessoas que apresentam sofrimento psíquico. Observa-se que apesar do conceito ampliado de Saúde ter quase 30 anos e ser oriundo de um grande movimento político (Reforma Sanitária), ainda existem discursos reduzidos a uma compreensão da Saúde como ausência de doença ou pleno bem-estar físico, mental e social.

Saúde mental é a pessoa que necessita de tratamento. Precisa de ajuda de outra pessoa pra poder fazer as atividades. (SI)

É complexo, sempre que penso em saúde mental, penso no conceito ampliado de saúde. Porque as pessoas lidam com os sofrimentos, as angustias, com os sentimentos de diversas formas [...]. (S5)

Scliar ${ }^{14}$ aponta que a Saúde reflete a conjuntura social, econômica, política e cultural. Não existe um conceito estático de Saúde, a significação difere de pessoa para pessoa e tem influências da época, lugar, classe social, valores individuais, concepções religiosas, entre outros aspectos.

Desse modo, observam-se transições do conceito de Saúde nas falas dos entrevistados. S1 utiliza a palavra "tratamento" a qual remete à doença e/ou cura, demonstrando o entendimento de Saúde como ausência de doença, com ênfase em aspectos biológicos, individuais e uma abordagem mecanicista. Ou seja, nesta compreensão de Saúde são desconsiderados aspectos sociais, econômicos e psicológicos do processo saúde-doença e, isto se amplia para o entendimento de Saúde Mental como psicopatologia e/ou tratamento de doenças mentais ${ }^{15,16}$.

Em outras falas se percebe um conceito de Saúde próximo ao conceito da OMS (1948), a Saúde perde o caráter negativo, mas, se coloca inatingível com o "completo estado de bem-estar". Contudo, em algumas colocações os entrevistados descreveram que existem momentos em que se vive aquilo que Canguilhem ${ }^{15}$ chama de "infidelidades do meio, fracassos, erros e o mal-estar" e isso também faz parte da Saúde, uma vez que não se pode pensá-la como ausência de sofrimento/ erros/decepções, mas, como um meio de enfrentar tais adversidades.

O que demonstra a passagem para o conceito ampliado de saúde, trazido na fala de S5, onde a saúde é vista como algo subjetivo, sujeita a modificações constantes e que é construída por momentos de sofrimento, prazer e pelas condições que permeiam a vida. Acredita-se que é nesse momento em que a Saúde Mental não pode ser dissociada da Saúde "geral”, coloca Amarante ${ }^{16}$, 
por mais que a Saúde Mental seja uma complexa área de conhecimento, é "um campo polissêmico e plural na medida em que diz respeito ao estado mental dos sujeitos e coletividades" (p.19).

Diante disso, há o reconhecimento de que as demandas em Saúde Mental estão presentes em diversas queixas que chegam aos serviços de Saúde, principalmente na APS, tornando-se um desafio de intervenção aos profissionais de saúde ${ }^{17}$.

Essas diferenças nas falas dos entrevistados indicam que ainda há formações em saúde pautadas no modelo hegemônico e com práticas reduzidas à medicamentalização e medicalização da vida. Barbosa ${ }^{18}$ potua que os próprios docentes notam que apesar das propostas de transformações na formação em saúde, ainda há "falhas", pois, os acadêmicos formam-se, mas, desconhecem "temas" relevantes para a prática profissional sendo um desses, a Saúde Mental.

\section{2 Vulnerabilidade social: quando se adoece "por" sociedade}

Ao analisar as falas dos entrevistados, nota-se estes relacionando o conceito de vulnerabilidade social a aspectos negativos, como pobreza, violência, falta de oportunidades e iniciativa. Afirmam Arregui e Wanderley ${ }^{19}$, é comum utilizar o termo vulnerabilidade para tais questões, assim como associá-la a exposição ao perigo e à insegurança social, colocando que o termo vulnerabilidade social parece surgir como substituto do conceito de pobreza.

Seria um nível de fragilidade psicológica, de não saber resolver. A falta de comprometimento com as coisas [...] é o financeiro, a escolaridade, o fator genético [...]. (S4)

São aquelas pessoas que não tem oportunidades na vida, às vezes, tem umas que não tem ânimo pra correr atrás das coisas, outras não tem oportunidades. (S8)

A maioria dos entrevistados demonstrou dificuldade em pontuar sua compreensão acerca da Vulnerabilidade Social, legitimando que se trata de um conceito complexo e com diferentes perspectivas. Existe uma parcela significativa de profissionais da saúde que não estão familiarizados com o conceito de vulnerabilidade, mesmo sendo um conceito antigo e historicamente ligado à saúde ${ }^{4}$. Esta dificuldade quanto ao conceito, pode reduzi-lo e/ou confundi-lo com pobreza (econômica), percebido na fala de um entrevistado ao se referir à vulnerabilidade social como aquela que se apresenta em famílias "realmente pobres", como se os conceitos fossem sinônimos.

Arregui e Wanderley ${ }^{19}$ discorrem que a percepção de pobreza como ausência de renda perdurou por anos e se legitimou apresentando um caráter multidimensional e vínculo com questões de desigualdades sociais. Embora se tenha discutido à proporção que a pobreza tomava, isto é, como carência de direitos, possibilidades e de acesso a serviços, quando eram exigidas medidas rápidas e efetivas para solucioná-la, a tendência permaneceu em reduzir a vulnerabilidade aos indicadores de renda. Essa restrição era justificada pela dificuldade na mensuração de aspectos considerados "inatingíveis" da pobreza, impossibilitando a comparação entre realidades. 
A vulnerabilidade se refere, segundo Ayres ${ }^{20}$ os "potenciais" de adoecimento ou não, relacionados a todo e cada sujeito que vive em certo conjunto de condições. Trata da possibilidade de deterioração no bem-estar como consequência da exposição ao risco, porém, dependerá dos recursos ativos e estratégias utilizadas para enfrentar situações externas ${ }^{19}$.

Apenas S4 relaciona a "responsabilidade individual" com a questão de "estar e/ou ser" vulnerável ao destacar que a Vulnerabilidade Social corresponde a "fragilidade psicológica". Isto é, as pessoas que se encontram nesta condição não estão "aptas" a solucionar seus problemas, "desesperam-se" e lhes "falta comprometimento com as coisas". Apreende-se que esse olhar pode se referir à culpabilização do sujeito, seja em relação a um adoecimento e/ou a uma condição da vida deste.

Ayres ${ }^{19}$ ressalta sobre o movimento de responsabilização do sujeito, pois, uma mudança comportamental não resulta apenas da informação e da vontade do sujeito. Mas, da disponibilidade de recursos culturais, econômicos, políticos, jurídicos que são desigualmente distribuídos entre os sexos, segmentos sociais, faixa etária, etc.

Observou-se, que ao mesmo tempo em que alguns entrevistados reduziam a vulnerabilidade social à pobreza, ampliavam-na quando se referiam as condições de vida. Tal pontuação vai ao encontro da fala de S8 quanto à falta de oportunidades cotidianamente enfrentada pelas pessoas, apontando a vulnerabilidade social como o modo como as pessoas privadas de seus direitos (saúde, educação, seguridade social, trabalho), expostas a riscos e com poucos recursos para alterar a dinâmica de suas vidas remanejam estratégias para defender suas condições de vida.

Para Hopenhayn ${ }^{20}$ vulnerabilidade e cidadania se mostram na evolução de questões sociais e se propagam como vinculadas ao acesso a direitos, emancipação e autonomia. Quando se pensa em vulnerabilidade, percebe-se uma "cidadania vulnerabilizada", uma vez que os atores sociais têm apresentado dificuldades em conciliar as vontades individuais com as coletivas.

As colocações dos entrevistados repercutem ao questionamento: como enfrentar situações de vulnerabilidade social? Ayres ${ }^{19}$ coloca que não são difundidos redutores desta questão, entretanto, pode-se questionar: o quanto os profissionais de saúde estão preparados para "provocar" essa mudança? O autor descreve que é preciso alertar sobre um "problema", mas, também proporcionar aos sujeitos meios de avaliarem e se instrumentalizarem para o enfrentamento de obstáculos materiais, culturais e políticos que os mantêm vulneráveis.

Esta questão relaciona-se ao "processo de conscientização", quando somente o homem é capaz de tomar distância frente ao mundo e agir conscientemente sobre a realidade objetivada. A conscientização implica em ultrapassar a esfera espontânea de apreensão da realidade para chegar a uma esfera crítica. Por meio desta unidade dialética, constitui-se o modo de ser ou de transformar 
o mundo que caracteriza os homens. Assim, a conscientização é inserção crítica na história, implica que os homens assumam o papel de sujeitos que (re)fazem o mundo, por isso, apresenta-se como um processo, "está" num determinado momento e no outro será de outra forma ${ }^{8}$.

Portanto, é preciso estar nesse processo de conscientização para conseguir transformar e encontrar estratégias de enfrentamento da vulnerabilidade social. Em relação ao enfrentamento, S5 mencionou capacidade de resiliência a situações de sofrimento psíquico e vulnerabilidade, e isso conduz a mais questionamentos: quem é ou está vulnerável?

Alguns filósofos apontam que a vulnerabilidade possui um sentido de condição humana, intrínseca ao sujeito e condição universal da humanidade 3 . O conceito de vulnerabilidade possui três características essenciais: multidimensional, em uma mesma situação os sujeitos estão vulneráveis a alguns agravos e noutros não; não é unitária, se está sempre vulnerável em diferentes graus; e não é estável, assim, as "vulnerabilidades" dos sujeitos alteram-se ao longo do tempo ${ }^{22}$.

Diante destas colocações, é possível refletir acerca da transformação das situações de vulnerabilidade social que os sujeitos se defrontam e, os profissionais de saúde ganham importante espaço nessa "luta" quando se tornam mediadores do encontro da população com o conjunto de recursos que a sociedade dispõe para construir a saúde. Contudo, não serão eficazes as tentativas caso permaneçam apenas na esfera da saúde, a ação intersetorial é essencial para mudança ${ }^{20}$.

O fazer dos profissionais de saúde tem que estar na transformação do cuidado em Saúde Mental, "ocupando-se" dos sujeitos e não de suas doenças. Isso não quer dizer que se ignora aquilo que é vivenciado pelo sujeito, mas, implica oferecer a este vez e voz para que deixe de ser invisível aos olhos da sociedade.

\subsection{De onde vem o sofrimento psíquico?}

Muitos são os aspectos que norteiam a vida das pessoas, questões culturais, familiares, políticas, direitos/deveres, possibilidades, trabalho, diferentes necessidades de autocuidado e lazer, etc. Tais esferas "compõem" as pessoas à medida que interagem com diferentes ambientes. Paralelamente, as esferas influenciam umas às outras e cada uma ao conjunto que é a pessoa ${ }^{17}$.

Alguns dos entrevistados pontuaram os fatores genéticos como influentes no sofrimento psíquico, enquanto a maioria trouxe aspectos relacionados ao ambiente, questões financeiras e relações familiares. Quando se fala em adoecimento humano não há como ignorar tais aspectos, principalmente, se as pessoas estiverem privadas de qualquer uma ou várias dessas esferas.

Anteriormente, ao referir problemas de ordem mental se utilizava a expressão "doença mental", "transtorno mental" ou "loucura". Contudo, a utilização destas expressões pode induzir as pessoas, inclusive os profissionais de saúde, a reduzir os problemas de Saúde Mental somente à 
causa genética e ao preconceito ${ }^{16}$. Ambas as situações limitam as possibilidades da vida das pessoas que vivenciam sofrimento psíquico.

As mudanças oriundas da Reforma Psiquiátrica não poderiam resultar apenas em modificações físicas, mas, numa reelaboração de concepções, ideais, maneiras de cuidar e assim, (re)construir e (des)construir os estigmas e preconceitos ainda existentes em relação a saúde mental ${ }^{23}$.

Fator financeiro, escolaridade, relacionamento na família. O fator preponderante é o grau de escolaridade, porque abre leque para essa pessoa não ter renda, se sentir impotente, deprimida, não ser uma pessoa de valor [...]. (S4).

Sempre defendi que a esquizofrenia tem um componente genético, mas, não é o determinante. Determinantes são as condições socioambientais, esse cenário dentro de casa é o determinante para o sofrimento [...] (S5).

A maioria dos profissionais de saúde apresenta, de certa forma, uma visão ampliada sobre os fatores que influenciam nos "problemas" de ordem mental, o que reforça a transição do conceito de saúde. Existem fatores estruturais (gênero, etnia, renda, escolaridade e trabalho) e fatores conjunturais (acontecimentos marcantes e o desencadeamento de certos sentimentos) que resultam em problemas de ordem mental ${ }^{16}$.

Com relação a essa visão ampliada, ainda longe de ser predominante, reflete-se que na $8^{\mathrm{a}}$ Conferência Nacional de Saúde, onde a saúde é entendida como resultante de condições coletivas de existência, como expressão ativa do exercício da cidadania ${ }^{24}$. Além de que, por meio desta, a sociedade tem a possibilidade de superar politicamente a compreensão vigente de saúde como estado biológico.

Percebe-se que não existe dificuldade apenas em conceituar saúde, mas, em discorrer os elementos que permitirão que esta seja assistida ou não. Badziak e Moura ${ }^{25}$ discutem a questão de Determinantes Sociais de Saúde (DSS), os quais se referem tanto aos comportamentos individuais quanto às condições de vida, por isso, pontuam que a relação dos determinantes com a saúde não é apenas de causa e efeito. Uma vez que estes não podem ser utilizados como "explicação" para todos os grupos de uma sociedade, pois, as diferenças existentes entre esses grupos são decorrentes das iniquidades sociais ${ }^{25}$.

Estas iniquidades sociais são percebidas pelos entrevistados, sobretudo os aspectos financeiros e escolaridade dos sujeitos, que as associam, ainda, à (im)possibilidade de aproveitar as oportunidades disponíveis na sociedade, correlacionando os dois aspectos ao "sucesso na vida". Logo, sem uma destas possibilidades as pessoas tenderiam a desenvolver sentimentos de frustração, incapacidade e inutilidade frente à situação vivenciada e esses sentimentos poderiam comprometer a saúde psíquica. 
Como pontuou S4 ao falar da relação entre escolaridade e as oportunidades que esta oferece, embora o sofrimento psíquico seja um fenômeno que não escolhe classe social, apresenta-se com maior amplitude em sujeitos vulneráveis, visto que estes tem menor possibilidade para melhorar as relações familiares e a qualidade de vida .

É interessante a colocação dos entrevistados acerca da influência que as relações familiares apresentam no sofrimento psíquico. Isso pode se dar pela crença cultural de que a família é a "base de tudo" e que através desta a transmissão de valores, normas culturais e o cuidado permitirá que seus membros se relacionem nos demais grupos. Contudo, quando a família enfrenta situações conflituosas e divergências de valores, pode resultar tanto em "problemas" de ordem mental, bem como aumentar o risco para eventos ameaçadores (delinquência, uso de drogas, etc.) ${ }^{6}$.

Sem contar que hoje a família tem enfrentado um momento de (re)estruturação, dando a impressão de desaparecimento desta ${ }^{26}$. Mas, na realidade se trata da mudança no tipo "prevalecente" de família, antes o patriarcalismo, hoje uma profunda diversificação. Devido a esta diversidade, observam-se mudanças na rede de apoio, aumento de lares madrecêntricos, sucessão de parceiros, etc. ${ }^{26}$, tais alterações no sistema de poder das famílias podem impactar no processo de saúdedoença-cuidado.

Além disso, Sluzki ${ }^{27}$ afirma que uma rede social pessoal estável é geradora de saúde, pois protege os envolvidos de doenças, acelera o processo de cura e aumenta sobrevida. Logo, se um destes membros adoece se espera que a rede social o apoie; entretanto, a presença de algumas doenças (sobretudo, as crônicas) produz um efeito de distanciamento em relação aquele membro que adoece e isso repercute na redução de contato social.

Nesse cenário, as redes sociais formadas dentro de outros contextos, por exemplo, serviços de saúde, ganham um caráter importante na recuperação e estimulação de habilidades sociais da pessoa em sofrimento. É perceptível o quão necessário se fazem estratégias e políticas públicas que considerem as principais influências sobre a saúde, pois, alguns comportamentos apresentam responsabilidade individual e requerem liberdade, no entanto, todo e qualquer comportamento é influenciado por determinantes sociais.

\subsection{A interface entre Saúde Mental e Vulnerabilidade Social}

Para a maioria dos entrevistados, Vulnerabilidade Social e Saúde Mental apresentam relação, mesmo que tenha havido dificuldade em descrevê-la. Apenas um profissional afirmou não perceber qualquer relação entre os conceitos.

Vai fazer a visita domiciliar e percebe que tem uma série de fatores sociais. Recurso financeiro escasso, a família que está cansada da situação, não é só a pessoa que tem a doença mental, mas, os familiares estão começando com algum sintoma e, geralmente, são as famílias mais empobrecidas (S2). 
É bem relativa, a gente vê pessoas que vivem bem, que tem tudo e apresentam uma porção de problemas de saúde mental. Podem acontecer problemas de saúde mental em pessoas mais vulneráveis, como em pessoas com um poder aquisitivo maior (S10).

Os entrevistados demonstram seguir a compreensão de vulnerabilidade relacionada à privação de recursos financeiros, de acesso à informação e que a vivência destas situações associada a questões de sofrimento psíquico conduzem a um agravo contínuo da Saúde Mental.

A família aparece, pontuada por S2, como "cuidadora" e, muitas vezes, tem sua saúde comprometida devido à situação de um dos seus membros (sofrimento psíquico) e também em consequência do contexto sociocultural que resulta em "sobrecarga" destes familiares. Esta sobrecarga decorre das dificuldades que os familiares encontram no desempenho deste papel importante e, por sua vez, pode ser acentuada pela ausência de apoio dos serviços e profissionais de saúde ${ }^{28}$.

A dificuldade de acesso ao serviço e às informações vai ao encontro do que se pontuou acerca da vulnerabilidade social. Ou seja, embora haja o compromisso profissional com o processo de desinstitucionalização e de fazer saúde humanizada, o apoio às famílias é fundamental. No entanto, mesmo que a família seja uma "peça" importante no processo saúde-doença, essa não possui o conhecimento e a preparação necessária para esse papel, ainda que a orientação, informação e suporte façam parte do processo de luta da Reforma Psiquiátrica (e também Sanitária) ${ }^{27 .}$

Diante disso, reflete-se: o que de fato afeta a saúde? Starfield ${ }^{29}$ faz uso do termo "condições de saúde" para explicar que a saúde é determinada pelas características ambientais, comportamentais e o senso de conexão e graus de coesão social da mesma. Além das condições sociais como, economia, educação, trabalho, oportunidades e outros aspectos disponíveis para as pessoas. Algo próximo do que S6 pontuou como sua compreensão da relação de vulnerabilidade e saúde mental, pois afirmou que quanto menos acesso o sujeito tiver, menor será sua qualidade de vida, bem como sua "estrutura" (psíquica) poderá ser reduzida no enfrentamento destas situações.

O não-acesso às condições de saúde diminui as chances de aquisição e aperfeiçoamento desses recursos, considerados fundamentais para que os sujeitos aproveitem as oportunidades oferecidas pelo Estado, mercado e sociedade para ascender socialmente. Por meio da vulnerabilidade é possível compreender "as vicissitudes e idiossincrasias existentes na realidade" ${ }^{30}$ dos sujeitos. Ao mesmo tempo, em que traz à tona o não-acesso aos insumos estratégicos e as consequências na condição de vida das pessoas (incerteza, insegurança, exposição ao risco), a vulnerabilidade consegue resgatar a disponibilidade de recursos e estratégias para que estas pessoas enfrentem as dificuldades que lhes afetam.

Entretanto, S10 afirma não perceber relação entre Saúde Mental e Vulnerabilidade Social, visto que os problemas ocorrem da mesma maneira em qualquer pessoa, independente da situação 
social vivenciada. Esta pontuação remete ao entendimento de saúde como ausência de doença e reduzido ao biológico, desconsiderando outras dimensões como influentes nos "problemas" de ordem mental.

\subsection{O (des)preparo profissional: a Saúde Mental como temática esquecida}

Os entrevistados percebem que os profissionais na APS não estão preparados para atuar com Saúde Mental. Alguns destacam que não houve, durante o período acadêmico, preparação para esta temática; outros relacionam com o interesse e a identificação do profissional com esta "área"; e também destacam a falta de conhecimento e o preconceito em relação aos usuários em sofrimento psíquico, como influentes neste (des)preparo profissional.

Não existe [preparo profissional], durante a graduação a gente não viu nada que se referisse a saúde mental. Para aprender a trabalhar foi no dia-a-dia [...] (S2).

Enxergo ainda $90 \%$ dos profissionais da saúde com estigmas para o atendimento em saúde mental. É mais fácil cuidar de uma ferida aberta, do que cuidar de um sofrimento psíquico [...] Estamos focados na queixa-conduta e, talvez, por desconhecimento (S5).

Com a implantação do SUS e a Reforma Psiquiátrica, o processo saúde-doença-cuidado passou a estimar os aspectos socioculturais, políticos e econômicos, superando [aos poucos] a compreensão de Saúde como ausência de doença. Mas, para que as transformações sejam legitimadas é importante que estas se estendam ao campo de formação de recursos humanos, considerada um dos entraves do SUS ${ }^{31}$.

Isto ocorre porque, por muitos anos, o modelo biomédico predominou e - mesmo após a readequação nas Diretrizes Curriculares Nacionais dos cursos de graduação em saúde, a qual propõe a formação de profissionais que devem proporcionar aos usuários atenção integral e humanizada ${ }^{32}$ - ainda ocupa espaço na formação e atuação em saúde. Diante disso, as falas dos entrevistados remetem ao questionamento sobre a atual formação dos profissionais de saúde. Para que as pessoas em sofrimento psíquico sejam reconhecidas como cidadãs e que os modos de cuidado e organização dos serviços confrontem as concepções tradicionais, exigem-se outros perfis profissionais. Na constituição destes perfis estão as universidades, que tem como desafio a formação em saúde conforme os princípios da Reforma Psiquiátrica ${ }^{31}$.

Ademais é interessante como alguns profissionais de saúde pontuam que existem duas áreas em saúde preparadas para a atuação em Saúde Mental, a Psicologia e a Psiquiatria, de certa forma, responsabilizam-nas pelo cuidado em Saúde Mental. Quando se fala em Saúde Mental, fala-se em história, em sociedade, em culturas e não apenas psicopatologias ${ }^{16}$. Por isso, a formação acadêmica precisa ser crítica, considerar a realidade e produzir novos conhecimentos e, em especial, articulada com uma atuação prática ${ }^{33}$.

\subsection{APS: ainda de portas semicerradas para Saúde Mental (e Vulnerabilidade Social)?}

ISSN 1982-8829 Tempus, actas de saúde colet, Brasília, 11(3), 50-68, 2017 - Epub mar, 2018 
Até pouco tempo trabalhar com Saúde Mental referia-se a hospícios, práticas desumanas, segregação, isolamento. Atualmente, os cuidados primários em Saúde tratam daqueles que são essenciais, imediatos, com ações que valorizam a realidade da comunidade e estão em alcance universal dos sujeitos que participam ativamente deste cuidado.

Em relação às principais ações dos profissionais da APS em Saúde Mental se elencam: detectar a queixa quanto ao sofrimento psíquico, oferecer escuta qualificada e proporcionar o cuidado adequado para situação ${ }^{34}$. Esta questão é trazida por S3 e S6; contudo, S3 pontua a necessidade de procurar auxílio da equipe para solucionar o "problema", enquanto S6 demonstra que há dificuldade nesta resolução e que existem profissões para determinados casos.

Acredita-se que essa aproximação da APS e Saúde Mental é também parte da Reforma Psiquiátrica, a qual não deve ser tratada apenas como surgimento de novos serviços para Saúde Mental, mas, como uma conquista e mudança de lógica assistencial. Este movimento inclui questionamentos acerca do modelo biomédico, participação da comunidade e, principalmente, a criação e diversificação de práticas e a ampliação de serviços ${ }^{35}$.

Contudo, como se evidencia nas falas da maioria dos profissionais de saúde, ainda a estratégia mais utilizada na APS é o encaminhamento. Acredita-se que essa lógica de encaminhamentos aos profissionais e/ou serviços compreendidos como especializados na área desconsidera a intervenção no âmbito da APS. Bem como, a Saúde Mental se torna "esquecida" na articulação com outras ações e políticas de saúde, quando esta deveria ser transversal em qualquer proposta de $\mathrm{Saúde}^{36}$. Deste modo, o cuidado em Saúde se torna mais compartimentado, tanto o atendimento quanto o sujeito.

O encaminhar, procurar a rede [...] A gente trabalha sempre junto, [...] a partir do que é decidido, monta uma estratégia, porque cada caso é um caso [...] (S3).

É bem deficiente essa parte [de estratégias] [...] A gente está acostumado a atender paciente na sua necessidade, quando foge dessa realidade a gente se sente deficiente [...] (S6)

De Vulnerabilidade Social só tem o matriciamento que deveria funcionar mais [...] Da saúde mental é aquele entrave, faz o diagnóstico ou tem o diagnóstico, só que não tem pra onde mandar [...] (S7).

Alguns profissionais referem, além do encaminhamento, que o psiquiatra terá melhor domínio e/ou apresentará uma conduta diferenciada em relação às pessoas em sofrimento psíquico. Entretanto, a APS apresenta uma possibilidade ampla para o cuidado em Saúde Mental. As ações desenvolvidas em um território geograficamente conhecido possibilitam aos profissionais que se aproximem da história de vida das pessoas, de seus vínculos com a comunidade onde moram, bem como com outros elementos dos seus contextos de vida ${ }^{17}$.

Percebe-se que os profissionais da APS formam-se ainda em um modelo hegemônico e suas

ISSN 1982-8829 Tempus, actas de saúde colet, Brasília, 11(3), 50-68, 2017 - Epub mar, 2018 
práticas, de modo geral, permanecem reduzidas à medicamentalização e medicalização da vida. Os profissionais referem dificuldades em lidar com o sofrimento psíquico, pontuando questões psicossociais destes sujeitos e o manejo de transtornos mentais 9 .

Através disso, fazem-se necessárias práticas que possibilitem e auxiliem os profissionais, principalmente na APS, a trabalhar com a dimensão do sofrimento psíquico. Pois, uma das fragilidades das equipes de ESF é o despreparo para lidar com situações de sofrimento psíquico, por mais que a Política Nacional de Saúde Mental preconize que os serviços realizem ações em todos os níveis de atenção à saúde ${ }^{37,19}$.

Em suas falas os entrevistados entendem que a família, muitas vezes, se abstém da responsabilidade de cuidado, especialmente após orientação da equipe de saúde. Acredita-se que isso decorre de um longo período onde o cuidado tinha como base o assistencialismo e, por vezes, ainda o é; o que resulta em usuários não autônomos e (des)responsáveis pela transformação de suas vidas.

Poucas foram as estratégias citadas pelos profissionais da APS em relação ao cuidado em Saúde Mental. Mencionou-se o matriciamento, que ocorre mediado pela equipe de NASF e envolve supervisão e capacitação da equipe de ESF no que se refere às "especificidades" dos profissionais do NASF. O matriciamento pode potencializar as equipes de ESF para atuarem em casos "difíceis", isto é, trata-se de uma troca de saberes, discussão e reflexão que antecedem as intervenções.

Toda e qualquer intervenção no sofrimento com vistas ao cuidado é terapêutica, pois o paciente, quando procura o serviço de Saúde, busca escuta, acolhimento, suporte e esclarecimento e isso também é terapêutico, independe do profissional ${ }^{38}$. Ou seja, através das equipes de ESF e NASF intensificam-se as necessidades de momentos para discussões, planejamento e definição de prioridades.

\section{CONSIDERAÇÕES FINAIS}

Observou-se, em consonância aos pressupostos dessa pesquisa, que o conceito de Saúde Mental se apresenta em transição. Ainda há profissionais de saúde que o limitam à psicopatologias, mas, há aqueles que o compreendem como "estado mental" sujeito à mudanças conforme as vivências, o tempo e o espaço. Alguns entrevistados trouxeram a necessidade de um cuidado pautado na escuta do usuário, respeitando as singularidades e possibilitando cuidado integral e promoção em saúde.

Embora os entrevistados salientem que se consideram (des)preparados para intervenções na APS quanto a Saúde Mental, esta visão sobre o cuidado é significativa, uma vez que indica que percebem a necessidade de (re)significar suas práticas e construir novos saberes. Porém, percebeuse que os profissionais, por não apresentarem uma formação acadêmica mais "específica" na área de 
Saúde Mental, não se sentem responsáveis quanto aos usuários em sofrimento psíquico. Reforça-se que se vivenciam momentos de fragmentação de conhecimento, mesmo com Políticas de Saúdes pautadas na interdisciplinaridade.

Esta fragilidade é também percebida na graduação que, apesar da reconfiguração das DCNs dos cursos da saúde, ainda forma profissionais para uma clínica segregada e biologicista. Tal formação acadêmica denota a falta de perfis profissionais "inquietos" com as incoerências e divergências na saúde coletiva brasileira. Pois, atualmente há políticas públicas que incentivam os profissionais (e os futuros) a estarem mais envolvidos e familiarizados com o SUS. Há oportunidades para ampliar a capacidade dos profissionais em manejar problemas de Saúde Mental na APS, contudo, o que os limita?

Diante desta reflexão, percebe-se que a prática de resgate e valorização da singularidade e dos direitos daqueles em sofrimento psíquico ainda se mostra escassa. A rede de cuidado em saúde não os percebe como sujeitos ativos e parece desconsiderar o lugar da cidadania no cuidado, restringindo-se a práticas de medicalização da vida.

O que remete à Vulnerabilidade Social, a qual os profissionais apresentaram dificuldade em descrevê-la, restringindo-a a pobreza. Mesmo com essa dificuldade, os profissionais de saúde desta pesquisa, demonstraram associar a Vulnerabilidade Social à falta de oportunidade frente às condições de saúde. Correlacionaram com as experiências cotidianas, onde os usuários dos serviços de saúde demonstram precisar de ajuda para remanejar estratégias em relação às vicissitudes da vida.

Através das colocações dos entrevistados, nota-se que veem relação entre Saúde Mental e Vulnerabilidade Social, pois, a exposição das pessoas a situações de Vulnerabilidade Social e risco de sofrimento psíquico, resultante de aspectos individuais, coletivos e contextuais, provocam maior suscetibilidade ao adoecimento e menor disponibilidade de recurso para se proteger ou reverter a situação $0^{20}$.

Não existem, ainda, estratégias que propiciem a conscientização das pessoas em sofrimento psíquico e Vulnerabilidade Social, para que estas assumam uma postura ativa e, assim, reivindiquem melhores condições de vida/saúde. Como questiona Ayres $^{20}$, quanto os serviços de saúde [e a rede de apoio] propiciam para que os contextos desfavoráveis sejam percebidos e superados pelos sujeitos? Possibilitam aos sujeitos transformar suas relações para se emanciparem das situações de vulnerabilidade?

Predominam estratégias higienistas sob roupagens de programas sociais e/ou serviços de Atenção em Saúde Mental, quando se precisa construir um novo modo de lidar com o sofrimento mental, acolhendo e cuidando dos sujeitos. A partir disso, construir-se-á um novo lugar social para a diversidade e o sofrimento mental ${ }^{16}$. 
Observa-se que alguns profissionais de saúde se "esquivam" dos aspectos significativos à saúde dos usuários. Portanto, é importante proporcionar momentos de reflexão - com rotinas de Educação Permanente - sobre o que os "incomoda" neste "tema". E assim, discutir e (re) construir, cotidianamente, o cuidado em Saúde Mental/Vulnerabilidade Social. Como apontam Abramovay; Castro $^{30}$, problemas como desigualdades sociais, discriminação, violência, ausência de condições mínimas e exclusão interagem entre si e formam "complexas redes causais" e, uma destas consequências é o agravo da Saúde Mental.

Acredita-se que esta pesquisa possibilita reflexões acerca das transformações que já ocorreram na Saúde Mental e dos desafios que prevalecem, ainda que tenha limitações como a não utilização de fontes secundárias para enriquecer a análise dos resultados. Principalmente porque algumas questões - como: por que os acadêmicos dos cursos de saúde se distanciam do SUS? Por que a resolução mais simples é a medicalização (e medicamentalização) extrapolada das "dores da vida"? - necessitam de respostas. Pois, enquanto o movimento for contrário aos "reais problemas" da sociedade, as consequências serão ainda mais "dolorosas".

\section{REFERÊNCIAS BIBLIOGRÁFICAS}

\footnotetext{
${ }^{1}$ Batista, AAG; Carvalho-Silva, HH. Família, escola, território vulnerável. São Paulo: CENPEC, 2013, 242p.

${ }^{2}$ Bezerra Júnior B, et al. Cidadania e loucura: políticas de saúde mental no Brasil. 7.ed. Petrópolis: Editora Vozes, 2001.
}

${ }^{3}$ Neves, MP. Sentidos da vulnerabilidade: características, condição, princípio. RBB 2006; v.2, n.2: p.157-172.

${ }^{4}$ Guareschi N; Bernardes, AG, et al. A Vulnerabilidade social e o programa Hospital-dia: uma discussão sobre as políticas públicas em Saúde Mental. Rev Cienc Humanas 2006; n.40: p.299-316.

${ }^{5}$ Padoin IG;Virgolin IWC. A vulnerabilidade social como uma dificuldade a participação política. In: XV Seminário Interinstitucional de Ensino, Pesquisa e extensão. Cruz-Alta: UNICRUZ, 2010. Disponível em http://www.unicruz. edu.br/15 seminario/seminario 2010/CCSA/A \%20VULNERABILIDADE $\% 20$ SOCIAL $\% 20 C O M O \% 20$ UMA $\% 20$ DIFICULDADE\%20A\%20PARTICIPA\%C3\%87\%C3\%83O\%20POL\%C3\%8DTICA.pdf Acesso em 18.05.2013.

${ }^{6}$ Grana L; Bastos AG. Vulnerabilidade Social: o psicodiagnóstico como método de mapeamento de doenças mentais. Psic. Ciência e profissão 2010; v.30, n.3: p. 650-661.

${ }^{7}$ Sánchez AIM; Bertollozzi MR. Pode o conceito de vulnerabilidade apoiar a construção do conhecimento em Saúde Coletiva? Ciênc e saúde coletiva 2007; v.12 n.2:p.319-324.

${ }^{8}$ Freire,P. Conscientização: teoria e prática da libertação - uma introdução ao pensamento de Paulo Freire. São Paulo: Cortez e Moraes, 2008.

${ }^{9}$ Brasil. Ministério da Saúde. Secretaria de Atenção à Saúde. Departamento de Atenção Básica. Diretrizes do NASF: Núcleo de Apoio a Saúde da Família. Brasília: Ministério da Saúde, 2010.

${ }^{10}$ Triviños, ANS. Introdução à pesquisa em ciências sociais: a pesquisa qualitativa em educação. São Paulo: Atlas, 2013. 
${ }^{11}$ Minayo, MCS. O desafio do conhecimento: pesquisa qualitativa em saúde. São Paulo: Hucitec. Rio de Janeiro. Rio de Janeiro: Abrasco, 2013.

${ }^{12}$ Lopes MJM; Leal SMC. A feminização persistente na qualificação profissional da enfermagem brasileira. Cadernos Pagu, 2005.

${ }^{13}$ Fernandes JS; Miranzi SSC, et al. A relação dos aspectos profissionais na qualidade de vida dos enfermeiros das equipes saúde da família. Rer Esc Enferm 2012, v. 46 n.2: 404-12.

${ }^{14}$ Scliar M. A história do conceito de saúde. Rev Saúde Coletiva 2007, v17 n.1:29-41.

${ }^{15}$ Batistella C. Abordagens Contemporâneas do Conceito de Saúde. In: FONSECA AF; CORBO AA (Org.). O território e o processo saúde-doença. Rio de Janeiro: EPSJV/Fiocruz, 2007. 266p.

${ }^{16}$ Amarante P. Saúde Mental e Atenção Psicossocial. Rio de Janeiro: Editora Fiocruz, 2015.

${ }^{17}$ Brasil. Ministério da Saúde. Secretaria de Atenção à Saúde. Departamento de Atenção Básica. Saúde Mental: caderno de Atenção Básica. Brasília: Ministério da Saúde, 2013. 176p.

${ }^{18}$ Barbosa TM; Melo A; Tonin CF. (Monografia) Os estilos de pensamento de professores da saúde sobre Saúde Mental. Trabalho de Conclusão de Curso apresentado ao curso de Psicologia da Universidade do Planalto Catarinense. Lages:UNIPLAC, 2012.

${ }^{19}$ Arregui CC; Wanderley MB. A vulnerabilidade social é atributo de pobreza? Serv Soc Soc 2009,n.96, p.143-165. ${ }^{20}$ Ayres JRCM, et al. Risco, vulnerabilidade e práticas de prevenção e promoção de saúde. In: CAMPOS, G.W.S. et al. Tratado de saúde coletiva. São Paulo: Editora Hucitec, 2012.

${ }^{21}$ Hogan DJ; Marandola Júnior E. Para uma conceituação interdisciplinar da vulnerabilidade. In.: CUNHA JMP (Org.) Novas metrópoles paulistas: população, vulnerabilidade e segregação. Campinas: Núcleo de Estudos de População, 2006.

${ }^{22}$ Ayres JRCM; França Júnior I, et al. O conceito de vulnerabilidade social e as práticas de saúde: novas perspectivas e desafios. In: CZERESNIA, D. ; FREITAS, C.M. Promoção de saúde: conceitos, reflexões, tendências. Rio de Janeiro: FIOCRUZ, 2014.

${ }^{23}$ Silveira LC; Braga VAB. Acerca do conceito de loucura e seus reflexos na assistência de saúde mental. Rev Latino-Am. Enfermagem 2005, vol13,n4.

${ }^{24}$ Amarante, P. Novos sujeitos, novos direitos: o debate em torno da Reforma Psiquiátrica. Caderno de Saúde Pública 1995. v11, n3: p.491-494.

${ }^{25}$ Badziak RPF; Moura VEV. Determinantes sociais da saúde: um conceito para efetivação do direito à saúde. R Saúde Pública 2010. v 3, n1.

${ }^{26}$ Castells M. O poder da identidade: a era da informação - economia, sociedade e cultura. São Paulo: Editora Paz e Terra S.A, 2001.

${ }^{27}$ Sluzki CE. A rede social na prática sistêmica. São Paulo: Casa do Psicólogo, 2006.

${ }^{28}$ Bandeira M; Barroso SM. Sobrecarga das famílias de pacientes psiquiátricos. J bras psiquiatr 2005. vol54, n1:3446.

${ }^{29}$ starfield B. Atenção primária: equilíbrio, entre necessidades de saúde, serviços e tecnologia. Brasília: UNESCO, Ministério da Saúde, 2004.

${ }^{30}$ Abramovay M; Castro MG, et al. Juventude, violência e vulnerabilidade social na América Latina: desafios para políticas públicas. Brasília: UNESCO, BID, 2002.

${ }^{31}$ Patriota LM. Saúde Mental, Reforma Psiquiátrica e formação profissional. In: Jornada Internacional de Políticas Públicas. João Pessoa: UFP, 2011. 
${ }^{32}$ Cavalheiro TP; Guimarães AL. Formação para o SUS e os desafios da integração Ensino e Serviço. Caderno FNEPAS, 2011.

${ }^{33}$ Ribeiro SL; Luzio CA. As diretrizes curriculares e a formação do psicólogo para a saúde mental. Psicologia em Revista 2008. v14, n2: p.203-220.

${ }^{34}$ Tanaka OY; Ribeiro EL. Ações de saúde mental na Atenção Básica: caminho para ampliação da integralidade da atenção. Rev Cienc e Saúde Col 2009,v.14,n.2:477-486.

${ }^{35}$ Souza AC; Rivera FJU. A inclusão das ações de saúde mental na Atenção Básica: ampliando possibilidades no campo da saúde mental. Rev Tempus Actas Saúde Col 2010. v.4 n.1:105-14.

${ }^{36}$ Silveira ER. Práticas que integram a saúde mental à saúde pública: o apoio matricial e a interconsulta. Ciência e Saúde Coletiva 2012. v.17.n.9: 2377-2386.

${ }^{37}$ Silveira DP; Vieira ALS. Saúde Mental e atenção básica em saúde: análise de uma experiência no nível local. Ciência e Saúde Coletiva 2009, v.14.n.1:139-148.

${ }^{38}$ Chiaverini DH (Org.). Guia Prático de matriciamento em Saúde Mental. Brasília, DF: Ministério da Saúde: Centro de Estudo e Pesquisa em Saúde Coletiva, 2011.

Artigo apresentado em 01/03/2017

Artigo aprovado em 16/10/2017 Artigo publicado no sistema em 16/04/2018 